\title{
Comparison of Light Pulses' Number Induced by Cosmic Radiations at Aircraft Flight Altitude using Experimental Data versus MCNP6
}

\author{
Sadia Assad ${ }^{1}$ \\ Department of Electrical and Computer Engineering \\ University of Quebec at Trois Rivieres \\ Trois Rivieres, Quebec, G9A H7, Canada \\ E-mail: sadia.assadeuqtr.ca
}

\section{Adam Skorek}

Department of Electrical and Computer Engineering University of Quebec at Trois Rivieres

Trois Rivieres, Quebec, G9A H7, Canada

E-mail: adam.skorekeuqtr.ca

\author{
Fidele Moupfouma \\ Department of Electrical and Computer Engineering \\ University of Quebec at Trois Rivieres \\ Trois Rivieres, Quebec, G9A H7, Canada \\ E-mail: fidele.moupfoumaluqtr.ca
}

\author{
Mohamed Al Skarkawy \\ Bombardier Aerospace \\ 2351 Saint Laurent Montreal, Quebec, H4S 1V3, Canada \\ E-mail: mohamed.elsharkawilaero.bombardier.com
}

The spontaneous assessment of the atmospheric cosmic radiations at aircraft flight altitudes is becoming important to airlines and certification authorities. The largest number of recorded fluxes is usually for neutrons and gamma rays. However, neutrons prove to be the greatest threat to the aircraft's electronic and electrical systems. In this context, Bombardier has launched a cosmic radiation measurement campaign, where plastic scintillators sensors are used. The use of plastic scintillators is governed by their robustness and their capability to discriminate between neutrons and gamma rays.

The sensor converts deposited energy levels, induced by cosmic radiations recorded into light pulses on-board flying aircraft. The MCNP 6 (Monte Carlo N Particles) software is then used with the appropriate modeling of the plastic scintillator to simulate the corresponding light pulses, which are then compared to the measured test data. All other testing parameters (i.e., altitude, longitude, latitude, and date) are taken in consideration during the simulation process. The scintillator geometrical model and the testing parameters referred to earlier are then used by the built in Function (+F6/DE/DF) in MCNP6 to compute the light energy mean number. Finally, to calculate the light pulses mean number, the following conversion rate of the scintillator was used ( 8600 photons/1 MeVee).

A mean relative error of $20 \%$ is deduced, when comparing the number of measured light pulses with the ones simulated with the MCNP6 software. Relatively, good agreement between both results is achieved, which falls within the acceptable practical tolerance.

36th International Cosmic Ray Conference -ICRC2019-

July 24th - August 1st, 2019

Madison, WI, U.S.A.

\footnotetext{
${ }^{1}$ Speaker

(C) Copyright owned by the author(s) under the terms of the Creative Commons

Attribution-NonCommercial-NoDerivatives 4.0 International License (CC BY-NC-ND 4.0). http: / / pos . sissa.it/
} 


\section{Introduction}

Nowadays, the aerospace industry achieves considerable advancement in research and development. As a result, flights over very long-distances at very high altitudes, flying over northern or southern latitudes become routine. Higher latitude and altitude deal with more radiative environment, meaning where aircraft system immunity could be affected.

In this context, IEC (International Electro-technical Commission) has published a series of guidelines and standards "IEC 62396" leading to the process of managing the avionicsAtmospheric radiation effects in order to help the aircraft's manufacturers and designers to avoid the occurrence of SEE (Single Event Effect) due to the atmospheric cosmic radiations [1].

In this perspective, Bombardier and its partners (CMC Electronics, BTI, ETS, StarNavigation, and ISONEO) have launched in the scope of CIMES project a measurement campaign of atmospheric cosmic radiations onboard a flight test aircraft, using a plastic scintillator associated to the adequate electronic system, in order to collect the light pulses number for the corresponding energies for all particles (with and without discrimination). This campaign serves to plan and to help the certification authorities in the preparation of future regulations to qualify and certify the avionic systems against cosmic radiations effects.

The measurement systems inside the airplane had been modeled in the cosmic radiations' environment by using the MCNP6 (Monte Carlo N Particle) software, to determinate the theoretical light pulses number for all particles.

\section{Atmospheric Cosmic Radiations}

Whether they come from other galaxies or they are of solar origin, the journey of cosmic radiations towards the earth atmosphere is carried out over very long distance. Consequently, their energy gets reduced gradually by many obstacles, that obstructed their routes, such as, IMF (inter planetary magnetic field), solar wind, and earth magnetic field. These lasted obstacles can deviate the cosmic radiations from their original routes (paths) to a far-away location from the earth [2].

Cosmic radiations are of galactic or extra galactic origin (GCR); and they can also be related to solar activity and be named (SCR), as presented in the Fig. 1. Both, can reach the earth atmosphere, however, the GCR presents $95 \%$ of the radiations intensity at the aircraft flying altitude. A great solar flare in 1956 had generated an increase in the radiation quantity of 300 times more than the nominal rate in few hours, for the high altitude and polar latitude [2-4].

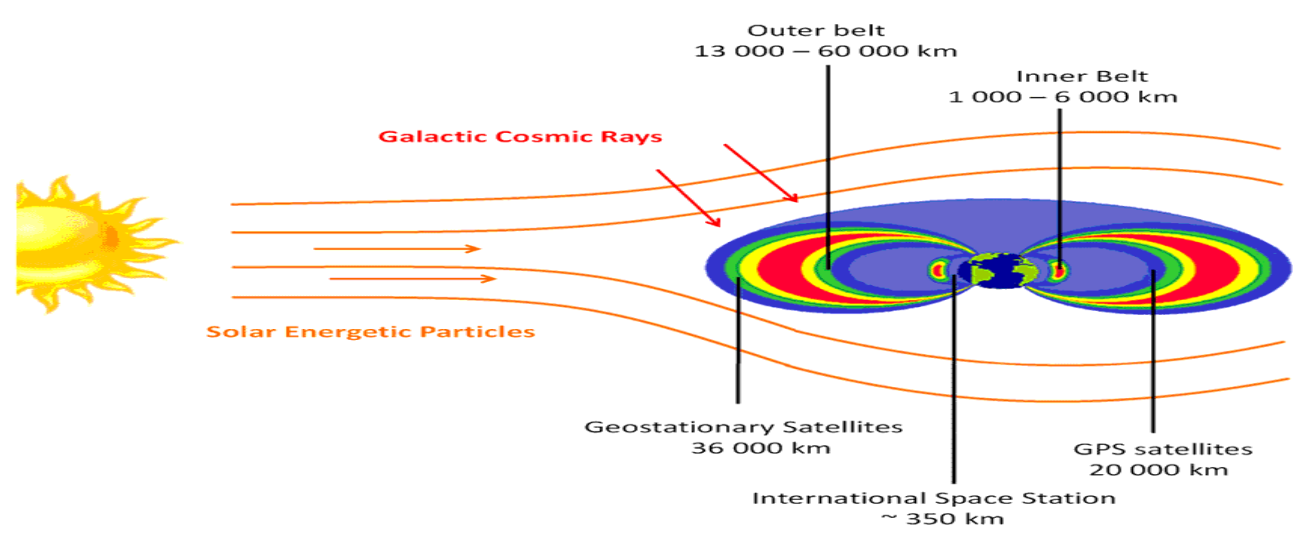

Fig. 1: Galactic and solar cosmic radiation with respect to earth [5]. 
The GCR are predictable and depend on the atmosphere thickness or altitude. This layer helps in reducing the radiations' flow. They also depend on the geomagnetic coordinates, which define the earth's magnetic field shielding intensity, and on the solar cycle. The solar cycle usually takes 11 years, which defines the solar wind and solar activity strengths. GCR are composed mainly of protons and helium with a maximum energy of $10^{20} \mathrm{eV}$ [2]. Their energy level at the top atmosphere layer of the earth reaches $10^{12} \mathrm{eV}$.

The SCR are non-predictable and are mainly composed of protons and electrons. This is due to the fact that they depend on the spontaneous and random occurrence of the solar flares. They also depend on coronal mass ejection "CME" of the sun [2].

The interaction of the primary particle cosmic radiations at the top layer of the atmosphere with its molecules, such as nitrogen and oxygen, makes a huge types of cascades that re generated along the expanse of the atmosphere until the energy of this cosmic particle runs out. These cascades are muonic, electromagnetics, and hadronic, as shown in the Fig. 2. The last two cascades are the most critical ones at the aircraft flying altitude, where gamma rays and neutrons are presented, respectively.

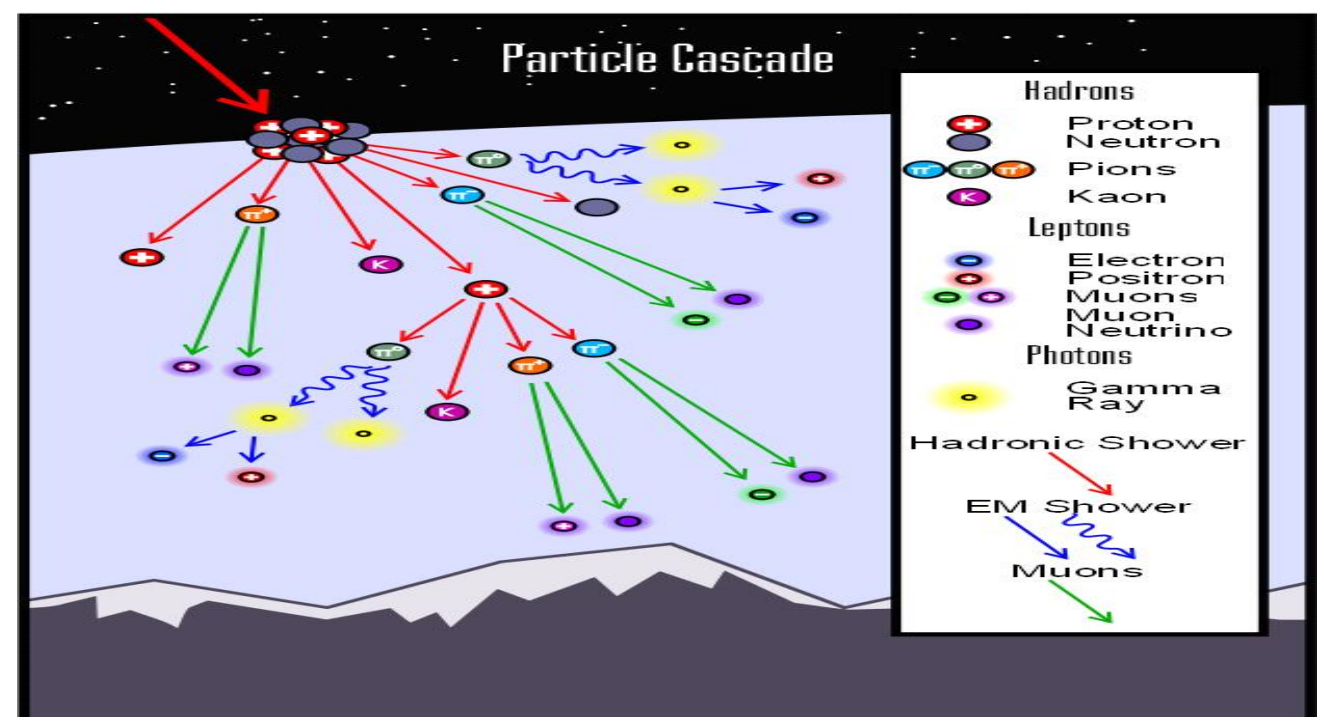

Fig 2. : Particles and showers cascades generated in the atmosphere [6].

\section{Process of Light Pulses Number Recording On-Board the Aircraft}

A cylindrical plastic scintillator (2" in diameter and 2" in height), as shown in Fig. 3, is used to detect and measure the cosmic radiation particles on-board of a flight test aircraft. This is because of its sensitivity to the fast neutrons and gamma rays through the production of the recoil protons and electrons, respectively. This happens after the interaction of the fast neutrons with hydrogen, which is the main component of the scintillator matter (see table 1), and the interaction of gamma rays with electrons. Furthermore, this type of scintillator is known for its capability to discriminate between the neutrons and gammas rays in the mix radiation field. A Photomultiplier (2" in diameter and 10 stages), as presented in Fig. 4, is used to convert the photons produced after the scintillation to photoelectrons through the photocathode semitransparent extended Bialkali. 


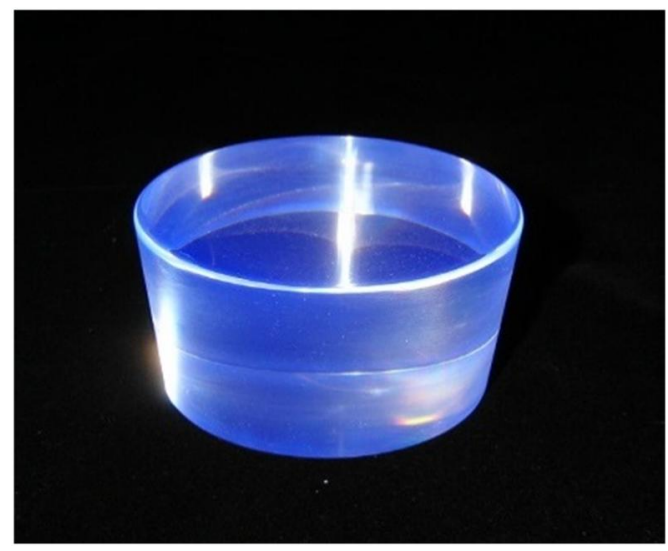

Fig. 3: Plastic scintillator: EJ 299-33A [7].

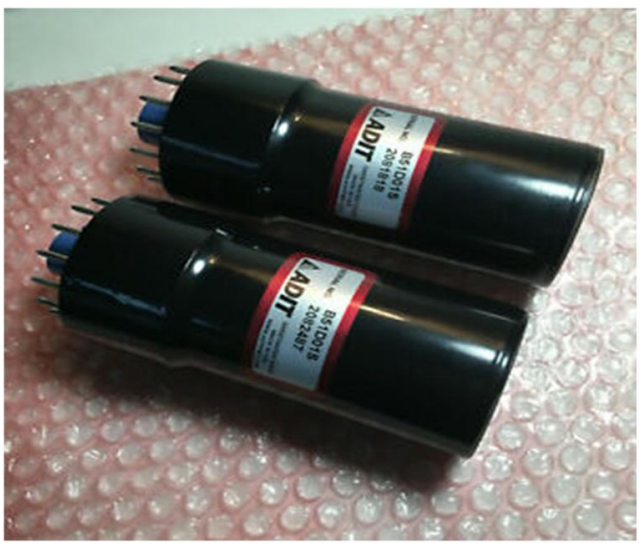

Fig. 4: Photomultiplier: Adit B51D01 [8].

Table 1: Scintillator proprieties.

\begin{tabular}{|c|c|}
\hline Proprieties & Scintillator \\
\hline Output Light (\% Anthracene ) & 56 \\
\hline Scintillation Efficiency & $\mathbf{8 6 0 0}$ photons/1MeVee \\
\hline Wavelength of Maximum Emission $(\mathrm{nm})$ & 420 \\
\hline H Atoms per $\mathrm{cm}^{3}\left(10^{+22}\right)$ & 5.13 \\
\hline C Atoms per cm $\mathrm{cm}^{3}\left(10^{+22}\right)$ & 4.86 \\
\hline E Atoms per $\mathrm{cm}^{3}\left(10^{+23}\right)$ & 3.55 \\
\hline Density $\left(\mathrm{g} / \mathrm{cm}^{3}\right)$ & 1.08 \\
\hline
\end{tabular}

An electronic system is used to record the pulses' histograms with and without discrimination. Fig. 5 shows an example to what can be used for the recording process, which is found in the literature [9] and is not explicitly mounted here. It employs the implemented algorithms in ADC (analogue digital converter) and FPGA (field programmable gate arrays). ADC allows the extraction of the best samples that are necessary for the FPGA. This is in order to provide, in real time, the number of light pulses with and without discrimination. This is done by observing the decay time difference between gamma rays and neutrons.

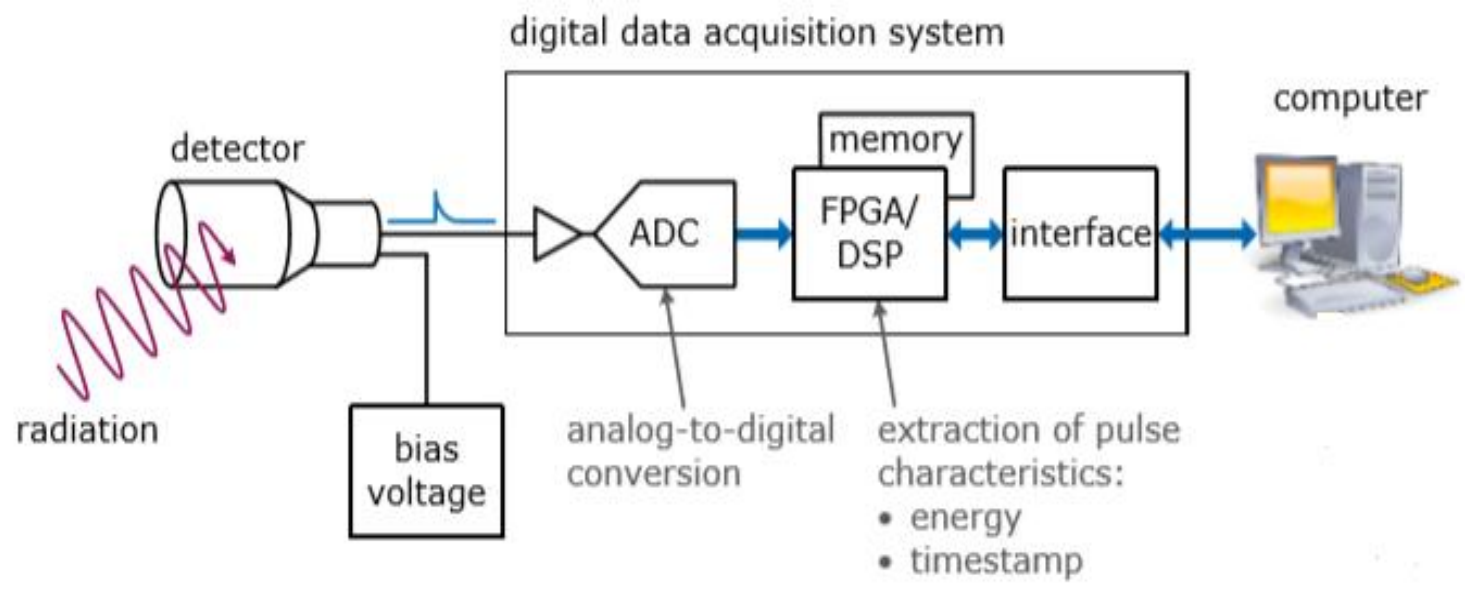

Fig. 5: Example of an electronic system prototype connected to the plastic scintillator [9]. 


\section{Tools for Atmospheriec Cosmic Radiations Assessment}

The primary cosmic radiation particles at the atmosphere's top layer, are mainly isotropic for almost all energies. There are two kinds of codes that can evaluate the shower's intensity of the secondary particles that were produced after the collisions of the primary particles and the atmosphere's molecules. Those two kinds of codes are deterministic and Monte Carlo codes. The deterministic ones use the Boltzmann's equations and assumptions. They have the advantage of converging in a fast rate to the anticipated output, which is related to the propagation of cosmic radiations through the atmosphere. However, they are not accurate. LIUN, HZETRN, and PLOTINUS, are the tools that use this type of coding. On the other side, the Monte Carlo codes track the primary particle from its interaction with the atmospheric molecules till the end of all the corresponding generated showers. They generate several simulations following a statistical study that represents the expected results. FLUKA, GEANT4, PHITS, and MCNP6 are the tools that use this type of coding [10].

\section{Modeling used to Calculate the Light Pulses Number in MCNP6 Software}

From section 4, MCNP6 (Monte-Carlo N particle) has been chosen mainly to calculate the energetic response matrix of the plastic scintillator for each particle. In order to quantify the cosmic radiations of all energy particles, both the MCNP6 response matrix and the collected measured data of each flight shall be mathematically deconvoluted. To achieve this objective, the detector model installed on the plane in the cosmic radiations environment should be simulated with MCNP6.

In order to validate the realized model using MCNP6, the present study which carries out the comparison of light pulses' number between the simulations and the measurements recorded on the detector, has been launched. MCNP6 allows to calculate the propagation of the cosmic radiations through atmosphere and also to simulate the interactions of these cosmic radiation particles with the detector's matter. This is completed by using Monte Carlo statistical methodologies and the incorporated cross sections. Moreover, the Monte-Carlo methods use sampling methods and probability laws to solve complex physical problems.

The geometry characteristics, including the scintillator's setup positioning within the aircraft, and the type of material of components constituting the scintillator and the airplane, should be integrated in the input file. The cosmic radiations environment is defined in the source part, within the same file. The source definition is divided into three parts: 1) particle type identification, using "par =cr", 2) solar cycle or activity identification, by using the adequate date in "dat", and 3) identification of longitude, latitude, and altitude goes through the filling of the part "loc". The height of the atmosphere is divided into a set of $1 \mathrm{~km}$ radius cylinders as shown in the Fig. 6. The density of air and the atmosphere thus constituent should be allocated for each cylinder. This is in order to maximize the efficiency of the modeling scheme [11]. 


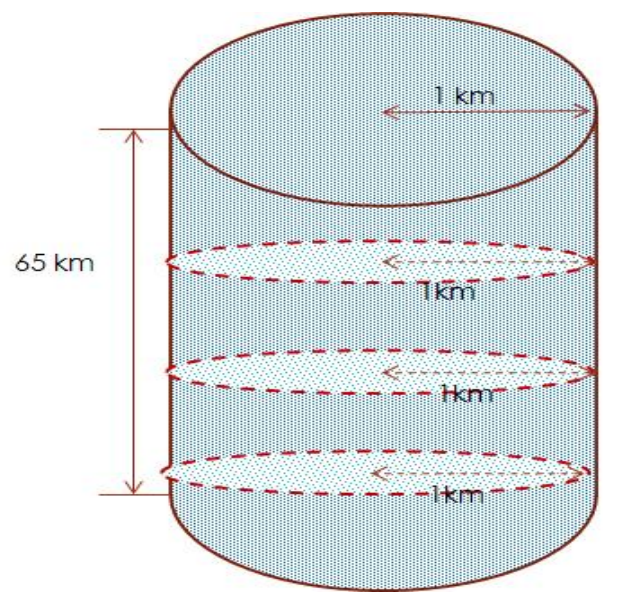

Fig. 6: Modeling of the atmosphere's height by the user.

In the scope of this work, the light produced by the interaction of all cosmic particles with the scintillator matter is calculated using MCNP6 software. The light pulses' number is assessed using the scintillator efficiency given in Table 1.

\section{Results and Comparison}

Some of the flight trajectory points are used in this study to compare the light pulses' number generated by all particles. Where the used light pulses' number are recorded, without discrimination in the electronic system, using the measurement system. On the other hand, the theoretical results are obtained following the simulation of the scintillator in Monte Carlo software. The modeling of the scintillator in MCNP6, is presented in Figs. 7 and 8.

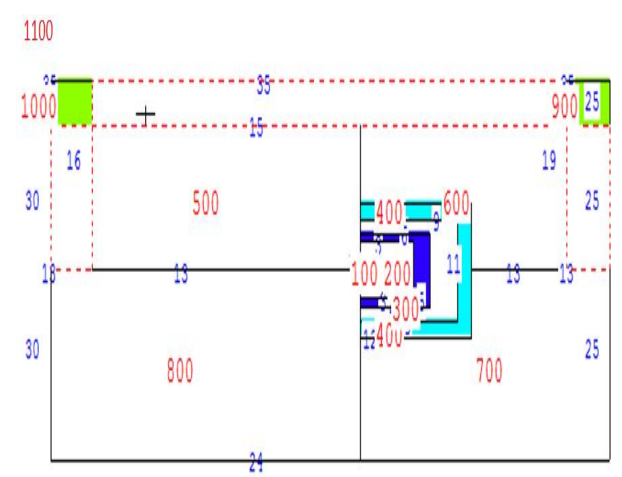

Fig. 7: 2D presentation of the Scintillator in MCNP6.

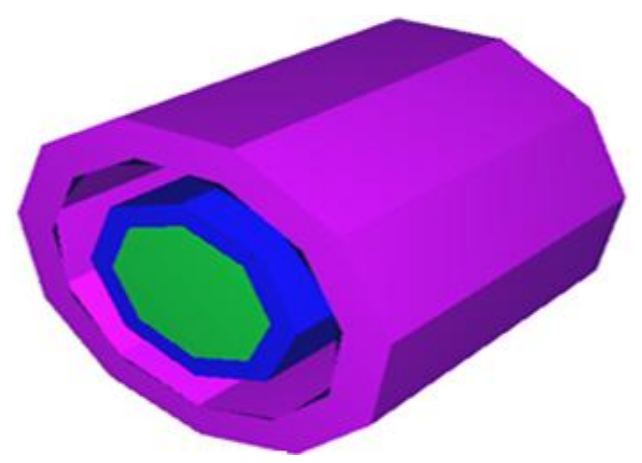

Fig. 8: 3D model of the Scintillator in MCNP6.

The simulation results in MCNP6 and the comparison of the light pulses number between the theoretical results and the experimental recordings are presented in Table 2 and Fig. 9. 
Table 2: Comparison of experimental and theoretical light pulses' number.

$\begin{array}{rrcr}\begin{array}{r}\text { Number of Flight Trajectory } \\ \text { Points }\end{array} & \begin{array}{c}\text { Theoretical Number of } \\ \text { Light Pulses }\end{array} & \begin{array}{c}\text { Experimental Number of } \\ \text { Light Pulses }\end{array} & \text { Relative Error }(\%) \\ 1 & 60 & 51 & 15.00 \\ 2 & 65 & 50 & 23.08 \\ 3 & 103 & 89 & 13.59 \\ 4 & 129 & 148 & 12.84 \\ 5 & 220 & 289 & 23.88 \\ 6 & 349 & 460 & 24.13 \\ 7 & 593 & 743 & 20.19 \\ 8 & 763 & 923 & 17.33 \\ 9 & 479 & 541 & 11.46 \\ 10 & 362 & 316 & 12.71 \\ 11 & 109 & 123 & 11.38 \\ 12 & 83 & 84 & 1.19\end{array}$

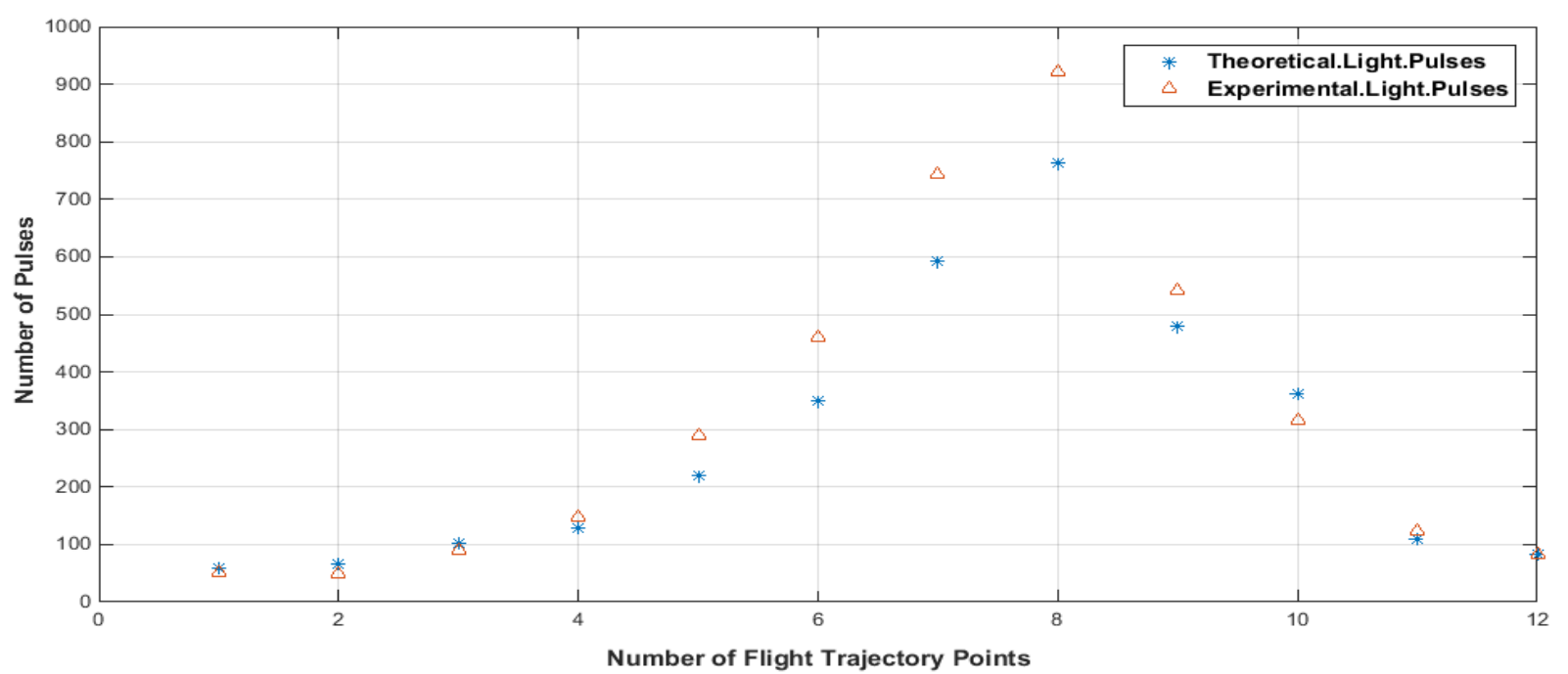

Fig. 9: Comparison of light pulses number induced by all cosmic particles.

\section{Conclusion}

The relative error between the theoretical and experimental results, doesn't exceed 25\%, which is acceptable in the detection and spectrometry of the radiation particles. It can then be concluded that model developed by using MCNP6 of cosmic radiations measurement's scintillator installed on board the test aircraft, provides good results compared to the recorded data. Therefore, it is promising to use the same model architecture to assess the energetic responses of this scintillator for each particle in MCNP6.

\section{ACKNOWLEDGEMENTS}

The authors would like to thank CRIAQ/CARIC, MITACS and Bombardier Aerospace for funding this research, as well as all the CIMES project partners 


\section{References}

[1] International Electro-technical Commission, Process Management for Avionics-Atmospheric Radiation, https://webstore.iec.ch .

[2] Office for Official Publications of the European Communities. Cosmic Radiation Exposure of Aircraft Crew: Compilation of Measured and Calculated Data.2004. ISBN 92-894-8448-9. p 281.

[3] L. O'.C. Drury, Origin of Cosmic Rays, Astro-Particle Physics, 2012. 39(1203.3681) 40: p.52-60.

[4] R. Edwards and al, Technical Standard for Atmospheric Radiation Event Effect (SEE) on Avionics Electronics, Proceeding in IEEE Conference Radiation Effects Data Workshop, DOI: 10.1109/REDW.2004.1352895

[5] H. Cottin and al, Space as Tool for Astrobiology: Review and Recommendations for Experimentations in Earth and beyond, 2017, Springer, p.100.

[6] K. Karkane, Cosmic Rays from the Telescope Array, 2013, The Telescope Array Collaboration, (https://astrobites.org/2013/06/04/cosmic-rays-from-the-telescope-array/ ).

[7] 2016 Eljen Technology, (https://eljentechnology.com/products/plastic-scintillators/ej-299-33a-ej299-34).

[8] ADIT, (https://radiadetecbus.com/b51d01s).

[9] J. Paepen and al, Characterisation of plastic scintillators used as an active background shield for neutron detection, 2016, JRC Technical Reports for European Atomic Energy Community, p.38.

[10] K A. Copeland, Cosmic Ray Particle Fluences in the Atmosphere Resulting from Primary Cosmic Rays Heavy Ions and their Resulting Effects on Dose Rates to Aircraft Occupants as Calculated with MCNPX2.7.0, 2014, Thesis presented to obtain a grade of Ph.D. at Royal Military College, Canada.

[11] Oak Ridge National Laboratory.MCNP6 Manual, 2014, Radiation Safety Information Computational Center «RSICC». 